\title{
Discordance dans l'expression des maladies liées au chromosome $X$ chez des jumelles monozygotes
}

Les anomalies cliniques dues à des affections génétiques liées au chromosome X sont en général l'apanage des garçons. Pour qu'elles se manifestent chez des filles hétérozygotes, il faut une inactivation systématique de l'X sain ; celle-ci peut se produire à la suite soit d'une anomalie chromosomique, soit d'un biais de l'inactivation d'un chromosome normal. Si la première explication est la plus fréquente dans nombre d'affections génétiques, c'est la seconde qui est en cause chez des jumelles monozygotes (MZ) dont une seule est atteinte d'une maladie génétique. Jorgensen et al. (Danemark et USA) [1] en font la démonstration à propos d'un trouble de la vision des couleurs : deux jumelles $\mathrm{MZ}$ étaient hétérozygotes obligatoires pour un gène de fusion vert-rouge, hérité de leur père atteint de deutéranopie (absence de vision du vert). Une des sœurs présentait l'anomalie, l'autre non. La sœur atteinte avait trois fils, dont un porteur du trouble visuel et deux sains. Dans les cellules cutanées obtenues par biopsie chez la sœur atteinte, presque tous les chromosomes actifs étaient d'origine paternelle, et d'origine maternelle chez la sœur indemne; en revanche, la proportion venant des deux parents était la même dans les lymphocytes circulants, probablement du fait d'une circulation fœtale commune.

Les auteurs notent que la discordance d'expression de caractères pathologiques chez des jumelles $M Z$ n'est pas exceptionnelle. Ils rappellent en outre l'exemple des célèbres quintuplées canadiennes Dionne, porteuses obligatoires d'un déficit de vision des couleurs, dont deux étaient daltoniennes, et dénombrent un cas de déficit en glucose-6-phosphate déshydrogénase, deux cas d'hémophilie $\mathrm{B}$, un cas d'X fragile et sept cas pour la vision des couleurs. Quant aux jumelles MZ discordantes pour la dystrophie musculaire de Duchenne (DMD), sept paires sont retrouvées dans la littérature (revue dans [1, 2]), dont quatre ont bénéficié d'une étude de l'inactivation de l'X. Les méthodes permettant de distinguer l' $\mathrm{X}$ actif de l'X inactif sont notamment fondées sur la différence du niveau de méthylation des deux $\mathrm{X}$. Ainsi, dans un cas, l'X de la jumelle cliniquement normale est inactivé au hasard, tandis que chez la jumelle myopathe, l'X sain est sélectivement inactivé. En revanche, dans les trois autres cas, l'inactivation préférentielle se fait en miroir. L'analyse en immunocytochimie de la dystrophine musculaire de ces enfants montre généralement des images normales de dystrophine chez les jumelles indemnes et, chez les jumelles atteintes, un certain pourcentage de fibres positives, probablement dû au caractère syncytial du tissu musculaire (quelques noyaux exprimant la dystrophine suffisant à rendre positive l'ensemble de la fibre).

Dans les cas d'X fragile où l'étude de l'inactivation a porté sur les lymphocytes, les jumelles présentaient une inactivation en miroir.

De toutes ces études ressort un élément biologique aussi intéressant qu'inattendu : alors que, dans 17 paires de jumelles $\mathrm{MZ}$ hétérozygotes pour une anomalie liée à l'X, une seule des sœurs est atteinte, on ne connaît aucun exemple d'une paire monozygote dont les deux soient affectées. Cette observation pose la question d'une relation causale entre gémellité $\mathrm{MZ}$ et inactivation préférentielle. Si la séparation gémellaire précédait l'inactivation d'un $\mathrm{X}$, celle-ci surviendrait indépendamment chez chaque jumelle, et la création systématique de types opposés serait très improbable. L'inactivation doit donc précéder la gémellité.

Pour expliquer l'inactivation en miroir, on pourrait imaginer qu'une répartition non aléatoire de l'inactivation de l'X au sein du blastocyste favorise la scission de l'œuf et soit ainsi responsable de la gémellité (la plus grande représentation du sexe féminin dans des paires de jumeaux attachés pourrait alors être due à l'apparition retardée de ce mécanisme).

Dans le cas où seule la jumelle atteinte inactive préférentiellement l'X normal, une autre hypothèse a été proposée : une scission asymétrique de l'œuf aurait pour conséquence le recrutement de cellules annexielles par l'embryon désavantagé (ce mécanisme a été observé lors de fécondation in vitro). Or, chez les mammifères étudiés, les cellules des annexes présentent une inactivation préférentielle de l'X paternel.

Aucune preuve expérimentale n'est venue à ce jour conforter l'une ou l'autre de ces hypothèses.

J.-C. D.

H. G.

1. Jorgensen AL, Philip J, Raskind WH, et al. Different patterns of $\mathrm{X}$ inactivation in $\mathrm{MZ}$ twins discordant for red-green color-vision deficiency. Hum Genet 1992 ; 51 : 291-8.

2. Gilgenkrantz S, Abadi N, Cheng M, et al. Mirror X-chromosome inactivation in female monozygote twins discordant for the clinical manifestation of Duchenne muscular dystrophy Tokyo : VIIth International Congress on Twin Studies, 22-25 June, 1992. 